\title{
Escenarios híbridos, narrativas transmedia, etnografía expandida
}

\section{Hybrid Scenarios, Transmedia Storytelling, Expanded Ethnography}

\author{
Daniel DOMÍNGUEZ FIGAREDO \\ Universidad Nacional de Educación a Distancia (UNED) \\ ddominguez@edu.uned.es
}

Recibido: 1 de febrero de 2012

Aceptado: 28 de mayo 2012

\begin{abstract}
Resumen
La transformación de los escenarios de socialización como consecuencia del impacto de las tecnologías digitales, introduce nuevas perspectivas para la investigación etnográfica. Tras superar los enfoques iniciales centrados en la dicotomía "espacios físicos-virtuales", se ha alcanzado una etapa de madurez que permite abrir nuevas vías conceptuales y analíticas para la metodología etnográfica aplicada en escenarios tecno-sociales. En este artículo se discute sobre la evolución de algunas dimensiones centrales de la etnografía de acuerdo con este nuevo marco social y epistémico. La discusión se basa en el análisis de las prácticas expandidas que tienen lugar en los nuevos espacios tecno-sociales, definidos como entornos de hibridación, donde las tecnologías están encastradas (embedded) en la vida física de los sujetos. Por un lado, se considera la producción de acciones basada en el ensamblaje de ideas, significados y objetos a través de dispositivos de mediación digitales. También se analiza su componente transmedia y las narrativas que permiten dotar de sentido a las experiencias. Como trasfondo del análisis, se introducen elementos para la discusión sobre el alcance de la investigación etnográfica expandida, la influencia del fenómeno transmedia en la noción de "campo" de estudio y los métodos que permiten determinar la significación a través de narrativas digitales.
\end{abstract}

Palabras clave: etnografía expandida, escenarios híbridos, narrativas transmedia, storytelling, protocolos simbólicos, ecosistemas abiertos.

\begin{abstract}
The transformation of social scenarios due to the impact of digital technologies, introduces new possibilities for ethnographic research. Once the initial approaches focused on the dichotomy of "physical-virtual spaces" have been overcame, it comes a stage of maturity that allows the ethnographers to open new avenues for conceptual and analytical methodology applied in techno-social scenarios. This article discusses the evolution of some key dimensions of ethnography according to the new social and epistemological framework. The discussion is based on the analysis of expanded practices that take place in the new techno-social spaces, defined as hybrid environments, where technologies are embedded in the physical life of the subjects. On the one hand, we consider the production of actions based on the assembly of ideas, meanings and objects through digital mediation devices. It is also analysed the transmedia component of the narratives that make sense to allow the experiments. Underlying the analysis, some elements are introduced for discussion on the scope of expanded ethnographic
\end{abstract}


research, the influence of transmedia phenomenon in the notion of "field" and the methods for determining the significance through digital storytelling.

Keywords: expanded ethnography, hybrid scenarios, transmedia storytelling, storytelling, symbolic protocols, open ecosystems.

Referencia normalizada: Dominguez Figaredo, D. (2012). Escenarios híbridos, narrativas transmedia, etnografía expandida. Revista de Antropología Social, 21: 197-215.

SUMARIO: 1. Introducción. 2. Tecnologías encastradas, capacidades amplificadas. 3. Etnografías de la hibridación. 4. Contextos ubicuos, cultura en red. 5. Prácticas expandidas, narrativas transmedia. 6. Referencias bibliográficas.

\section{Introducción}

La etnografía es la principal herramienta metodológica empleada en el análisis y la comprensión de los fenómenos socioculturales desde un enfoque naturalista ${ }^{1}$. En su versión actual, hacer etnografía consiste en un proceso continuado de aproximación a los agentes sociales y de recopilación de evidencias contextuales de diferente tipo con las que plasmar los rasgos de una determinada cultura. Sin embargo, el proceso hasta alcanzar esa caracterización y su "estatus" privilegiado como método reconocido no se ha producido de modo lineal y continuo.

La centralidad metodológica de la etnografía no ha impedido que ciertos aspectos relacionados con sus procedimientos, con el rol de los agentes que la llevan a cabo, con los contextos donde se desarrolla o con la validez misma de sus aportaciones, hayan estado sometidos a crítica y debate desde sus inicios. En las últimas décadas se han producido episodios de crisis más o menos profundas, motivadas principalmente por las aportaciones de otras tradiciones de pensamiento como el constructivismo, la fenomenología y la hermenéutica, que llevaron a poner en cuestión buena parte de sus postulados ${ }^{2}$. Superadas esas discusiones, la reflexión sobre el hacer etnográfico cobra ahora un nuevo protagonismo a propósito de la "consolidación digital", que podría definirse como la etapa sociohistórica que surge tras superar el primer impulso que supuso la aparición de la web y la apropiación social de las funcionalidades asociadas a esta tecnología. Pues bien, lejos de ser laterales, estos nuevos replanteamientos derivados de lo digital están suponiendo "otra vuelta de tuerca" sobre las ya clásicas reflexiones en torno al fondo y a la forma de hacer etnografía (Díaz de Rada, 2010).

Un rasgo que ha caracterizado la evolución de este método en los últimos años — etapa en que las tecnologías digitales se constituyen en el estándar de la mediación

${ }^{1}$ El término "naturalista" se emplea aquí de un modo genérico, con independencia de matices y revisiones (ver Hammersley y Atkinson, 1994).

${ }^{2}$ Debido a su interés analítico en el marco de este artículo, recordamos las tres crisis más destacadas de la etnografía, a saber, de la representación, la legitimación y la praxis (Denzin, 1996), así como algunas de las causas que las motivaron, como son, los cambios disciplinarios en la antropología social, la extensión del método a todo el amplio campo de las ciencias sociales y la tendencia creciente a trascender en ámbitos aplicados (Velasco y Díaz de Rada, 2006). 
comunicativa-, es la proximidad entre los debates que se centran en el desarrollo y los cambios metodológicos y aquellos otros ocupados de analizar la revolución digital como fenómeno, estos últimos procedentes en su mayoría de la sociología y la antropología social. De manera que se puede afirmar que las principales reflexiones sobre el papel de la etnografía en/de Internet y en el terreno de la mediación, han transcurrido últimamente en paralelo a las derivas tanto conceptuales como aplicadas surgidas a partir de las transformaciones en la estructura social y la cultura como consecuencia de la digitalización ${ }^{3}$.

Puede decirse pues, que el análisis sobre cómo se redefinen las prácticas socioculturales cuando se ven afectadas por el impacto de lo digital y, de rebote, cómo eso afecta a la etnografía, ha sido una constante practicada desde las disciplinas que recurren habitualmente a este método. La literatura sobre esas reflexiones se remonta a los primeros usos sociales e instrumentales en la investigación cualitativa de las tecnologías digitales (Allen, 1996; Biella, 1996; Bijker y Law, 1992; Dicks y Mason, 1998; Farnell y Huntley, 1995; Gray, 1995; Haraway, 1991; Markham, 1998; Meyrowitz, 1985; Woolgar, 1996), tiene un momento de eclosión en la penetración masiva de la web y su tratamiento como artefacto capaz de plasmar la cultura en la sociedad de la información (Beaulieu, 2004; Herring, 2004; Hine, 2000, 2005; Howard, 2002; Illingworth, 2001; Leung, 2005; Mayans, 2002; Miller y Slater, 2000; Silver, 2004; Woo, 2003) y, como mostraré más adelante, en el presente continúa de la mano de los debates sobre la hibridación sociotécnica, la conectividad y su aplicación en las prácticas cotidianas (Ardévol, Estalella y Domínguez, 2008; Burrell, 2009; Hine, 2007).

Resulta bastante evidente el paralelismo entre la evolución de la etnografía digital y de/en Internet y otros estudios sobre qué es "lo digital", sobre los usos y el impacto social de las tecnologías asociadas a ese fenómeno y, en general, sobre la influencia de los nuevos medios en las sociedades contemporáneas ${ }^{4}$. Como es propio, la relación estrecha que se ha dado entre las reflexiones meramente etnográficas y otras de tipo socio-antropológico ha generado múltiples efectos. Uno de los más destacados es el que lleva a enfatizar el componente procedimental de la investigación, que afecta básicamente al "cómo" se desarrolla la investigación etnográfica con nuevas tecnologías e Internet. De alguna manera, el proceso - repetido también en otras áreas científicas, cuando se trata de introducir la dimensión

${ }^{3}$ En el caso específico del método etnográfico, las diferentes aproximaciones se sintetizan en la dicotomía según la cual Internet puede concebirse como objeto de estudio o instrumento para el trabajo de campo de otros. Según Estalella y Ardévol (2010: 1), habría dos aproximaciones distintas: "la primera concibe Internet como una herramienta de investigación para aplicar determinadas técnicas (entrevistas, cuestionarios, etc.); la segunda plantea Internet como un campo de estudio".

${ }^{4}$ Las reflexiones sobre "lo digital" desde el campo de la sociología y la antropología social, han generado múltiples debates sobre la materialidad y los límites del ciberespacio como un entorno para la práctica y la negociación de significados. De manera que, como sucediera en las etapas anteriores, también en esta ocasión las cuestiones que son del interés de las ciencias alrededor de la etnografía parecen haber condicionado directamente el desarrollo y la validez de la metodología, aplicada ahora en ecosistemas digitalizados. 
digital — consiste en analizar cómo afecta cada nueva tecnología en el quehacer del etnógrafo y plantear a continuación las derivadas de los nuevos formatos que ahora son posibles en los procedimientos habituales del método.

En parte debido a esa circunstancia y en parte también por la presencia de otros inhibidores, como puede ser la dificultad de generar certidumbres en el escenario digital $^{5}$, han sido menos habituales las reflexiones sobre el cambio que introduce lo digital en otros componentes de la etnografía igualmente sustantivos, como sería el caso de sus bases epistémicas. Lo cierto es que, pese a constatar que no ha habido un cambio aparente en el marco de intenciones de la etnografía, sí es posible percibir algunos elementos novedosos que afectarían a la constitución de la disciplina como consecuencia de las posibilidades que surgen con los escenarios digitales. Y de ese modo, el avance en formatos, instrumentos y técnicas derivado de la digitalización hace que sea de interés plantear cómo afecta este escenario de prácticas mediadas ya consolidado a algunos rasgos epistémicos característicos de la etnografía ${ }^{6}$. En el siguiente apartado nos detendremos en los avances producidos específicamente en dos de esos rasgos, relacionados con la producción de contextos y con la forma de ensamblar relatos y generar patrones culturales. Y también en cómo su desarrollo abre nuevas vías al ejercicio etnográfico.

\section{Tecnologías encastradas, capacidades amplificadas}

Desde un punto de vista analítico, los medios digitales constituyen espacios públicos que complementan el medio físico donde se conforma la sociabilidad cotidiana. La estructura de ese espacio público no es estática, sino que avanza en función de las interacciones complejas que se dan entre sus dos componentes esenciales: el tecnológico y el social. A su vez, de la interacción entre esos componentes resulta una estructura tecno-social compleja, que presenta como principal rasgo característico la hibridación de las tramas sociales 'online' y 'offline'. Ese factor híbrido afecta directamente a la estructura de la sociabilidad, provocando cambios significativos en el comportamiento de los sujetos y nuevas formas de definir las comunidades y sus productos culturales (Domínguez y Álvarez, 2012).

La situación descrita supone una evolución radical en lo relativo a algunas concepciones anteriores sobre la posibilidad de aproximarse a los medios digitales y a los intercambios comunicativos en su "interior" desde enfoques parciales. En un escenario híbrido no es posible analizar las prácticas socioculturales atendiendo solo una de sus dimensiones analíticas, como pueden ser la parte online o la offline. Un

${ }^{5}$ Una característica distintiva del escenario digital es, justamente, la constante innovación en su marco de acción que es fruto de un proceso de intercambio bidireccional e iterativo entre el conocimiento técnico y el derivado de los usos sociales de la tecnología (Castells, 2001).

${ }^{6}$ Hablar de mediación significa señalar las transformaciones que se producen cuando objetos e individuos entran en relación (Callon, 1986; Latour, 1994). Por su parte, hablar de prácticas mediadas tecnológicamente significa considerar la producción de realidad que se genera en el marco de la interacción entre sujetos y aparatos (o 'software') como algo más que rasgos representativos de esa realidad. En ese sentido, por ejemplo, "un blog no es solo un conjunto de 'textos' que deben ser interpretados, sino un objeto que media en la interacción social entre individuos, y entre estos y el etnógrafo" (Ardévol, Estalella y Domínguez, 2008, xx). 
posicionamiento de ese tipo sería contrario a algunos principios epistémicos de la etnografía como el holismo y la intersubjetividad, y confrontarían con el propio significado del constructo "comunidad" (Domínguez, 2007). A lo sumo, las aproximaciones parciales de ese tipo pueden dar como resultado etnografías de alcance limitado o deficitarias respecto de lo que sería una deseada aproximación multi-contextual a la comunicación entre personas, que es la propia de los métodos naturalistas.

Por otro lado, la necesidad que tiene la etnografía de superar las constricciones de los enfoques parciales está también relacionada con el proceso crecientemente expansivo que se siguió para plasmar las prácticas sociales y las temáticas tratadas en los estudios sobre entornos mediados digitalmente. En los momentos iniciales del desarrollo de las redes y los dispositivos de comunicación, tanto las herramientas como el contenido de las conversaciones se limitaban al desarrollo de los protocolos, el software o los movimientos organizativos a su alrededor que posibilitó el surgimiento de Internet. La estructura de esa conversación y sus productos culturales asociados han sido ampliamente estudiados desde múltiples disciplinas del ámbito de las ciencias sociales, dando lugar a numerosos estudios sobre las comunidades descentralizadas de desarrolladores de código fuente. Asimismo, también se generó abundante literatura sobre cuestiones tecno-sociales, en lo que podría denominarse una primera generación de estudios sobre ciencia, tecnología y sociedad en las redes digitales?

Una resultante destacada de ese proceso es la que ha llevado a crear una cierta mitología sobre las propiedades constitutivas de los agentes de la acción en el mundo digital que son responsables del devenir de las redes. Algo que también ha llevado a establecer un alto grado de endogamia analítica, unido a una cierta seducción por el dominio de las competencias tecnológicas propias del mundo de la informática. De modo que, en ocasiones, se ha asumido que el análisis y la interpretación de las acciones mediadas digitalmente se circunscribe a los protocolos de comportamiento específicos de cada medio. Con lo que hacer investigación cualitativa en/sobre un medio digital demandaría del investigador poseer los conocimientos técnicos suficientes para actuar en el contexto donde tienen lugar las prácticas organizativas y comunitarias en ese medio ${ }^{8}$.

${ }^{7}$ Para una primera aproximación a la lógica de las comunidades virtuales previa a la eclosión de las redes sociales, véase Rheingold (1993), Turkle (1995), Jones (1998), Di Maggio et al. (2001) y Katz et al. (2001). Un análisis genérico de las temáticas de los movimientos sociales en la web de primera generación puede verse en Diani (2000). Puede verse un análisis sobre cómo la arquitectura tecnológica condiciona las prácticas sociales en la web en Mayans (2001), en el caso de los canales de Chat IRC, y Estalella (2005a; 2005b), en el caso de las comunidades de 'bloggers'.

${ }^{8}$ La reflexión en este caso no afecta a la necesaria inmersión del etnógrafo en el campo, y la oportunidad de conocer las bases culturales de los grupos que está analizando, como es el caso del idioma, sino que más bien se trata de poner en cuestión el requerimiento, por indicar un ejemplo prototípico de las primeras etnografías de Internet, de disponer de conocimientos de programación en determinados lenguajes informáticos para poder investigar las prácticas de mediación comunicativa en un grupo de desarrolladores colaborando en el diseño de una aplicación web. 
Una vez superadas estas aproximaciones restringidas al fenómeno de lo digital, se ha dado paso a formas de relación mediadas más abiertas, asumiendo que las tecnologías están presentes de manera indirecta, encastradas (embedded) en la vida física de las personas, y que los contenidos y las prácticas cubren todo el espectro temático de la cotidianidad. Es en ese sentido que también la práctica etnográfica se ha visto afectada directamente por la penetración de la tecnología digital, tanto en el quehacer del investigador como en los objetos de su estudio.

Las tecnologías digitales se asientan en la trama social y, una vez que han sido apropiadas por los usuarios, contribuyen a redefinir los mismos protocolos que regulan los comportamientos sobre la base de la expansión de las capacidades. Ese componente expansivo y otras cuestiones atribuidas específicamente a los entornos web, como la proliferación de sitios abiertos, las técnicas de analítica de datos masivos o la computación en la nube, han situado al investigador y al método etnográfico ante un escenario novedoso que amplía las bases de su actuación.

A propósito de la noción de expansión, es interesante señalar que cuando hablamos de prácticas expandidas - ya sean realizadas por el etnógrafo o por los sujetos de investigación - nos referimos a un tipo de actividad social que se proyecta más allá del entorno físico donde los individuos inician su acción. Por su parte, las prácticas expandidas mediadas tecnológicamente estarían remitiendo a las condiciones de libertad de las personas de actuar en otros entornos diferentes del físico.

La condición de "expansividad" no se centra pues en el efecto mismo de la acción y su concreción en situaciones distantes, ya que esto es en si evidente - en algunos casos es además una constante, como sucede en la comunicación mediada por tecnologías: cuando una persona habla por teléfono con otra, la construcción de significados se produce en un escenario diferente al de los emisores- - . La expansión se entiende aquí en el sentido de potenciación de las capacidades de acción del individuo en diferentes ambientes y sobre múltiples agentes de modos distintos. Incluso se trata también de una expansión temporal. Una auténtica redefinición de la topología espacio-temporal de los agentes.

Esta concepción entronca y se inspira en el enfoque de Amartya Sen', para quien la capacidad representa la libertad de una persona para elegir opciones alternativas de hacer y de ser. Esa capacitación se desarrolla en determinadas combinaciones de "funciones", que representan las cosas que pueden hacerse. De acuerdo con este enfoque, la expansión de las prácticas se entiende como un incremento directo de las posibilidades de los sujetos de operar en múltiples contextos de acción, lo que redunda en su mayor capacitación.

Las tecnologías digitales introducen nuevas funcionalidades, mejorando las condiciones de posibilidad para que se produzca dicha capacitación. Es en ese sentido que la mediación digital genera prácticas expandidas que permiten procesos, formatos, finalidades, estructuras que amplifican las realizadas en entornos analógicos.

${ }^{9}$ Para un análisis detallado de la noción de expansión de las capacidades en Amartya Sen, acompañado de una amplia revisión bibliográfica de su obra, véase Álvarez (2001). 
Situando el análisis en el marco de la etnografía, tenemos que ésta ve incrementado su potencial cuando se aplica en contextos tecnosociales. Por un lado, refuerza su dimensión holística, permitiendo incorporar al relato etnográfico elementos descriptivos y argumentales en planos de la realidad más allá de lo físico. También se da una expansión en el ámbito aplicado, abriendo nuevos contextos de estudio en una variedad de situaciones. Si entendemos el contexto como el ámbito que permite plasmar el significado de un fenómeno social y tomamos en cuenta su vinculación con la noción de trama etnográfica (pattern), entonces es posible inferir un conjunto de nuevas relaciones que se producen como consecuencia de sumar nuevas capas de sociabilidad a los grupos y situaciones que son objeto de análisis. Esta circunstancia no solo afecta a las metodologías de carácter intensivo como la etnografía, sino que impacta igualmente en otras estrategias de investigación extensivas. Si bien es en los casos en los que prima la acotación temporal y geográfica donde el cambio de orden se pone especialmente de manifiesto.

En relación con el contexto, cabe decir pues que el significado que adquiere este constructo en un escenario hiperconectado - donde la suma de los nodos que lo constituyen es siempre inferior a su capacidad de influir en las prácticas de los grupos que lo habitan - es una cuestión que afecta a algunos principios básicos de la etnografía. Los más directamente implicados pueden ser su dimensión holística y la representatividad de las aportaciones del investigador. Por ello, puede resultar de interés detenerse a continuación en los aspectos estructurales y sistémicos (en términos más dinámicos) que definen el contexto de acción del etnógrafo y las formas en que se plasman las relaciones en los escenarios de práctica digitales.

\section{Etnografías de la hibridación}

Etnografía virtual, ciberetnografía, webgrafía, etnografía en la(s) pantalla(s), etnografía de la mediación, etnografía 2.0, wikietnografía, etnografía P2P... Existen múltiples calificativos para definir los modelos de etnografía que surgen con la proliferación de nuevos espacios digitales. Esa polisemia terminológica puede explicarse en el marco de un cierto determinismo en favor de la infraestructura digital, algo que también es habitual en otras disciplinas y que se debe, en buena medida, a la capacidad de esas tecnologías de impactar en todos los órdenes de la sociabilidad $^{10}$.

En el caso del método etnográfico, detenerse en los motivos que explican ese mapa conceptual proporciona algunas derivadas de interés. En un principio, no resulta extraño ni novedoso que las definiciones de la etnografía carguen la significación en los elementos infraestructurales de una determinada cultura, llegando a apropiarse de los adjetivos que la definen (como, por ejemplo, cuando se emplean términos como e-etnografía o ciberetnografía). De hecho, es propio del relato

${ }^{10}$ Esa variedad de descriptores ha generado un amplio ecosistema metafórico que afecta igualmente a otras muchas disciplinas (Gómez, 2007). Por norma, cuando una acción social puede hacerse mediada a través de la web, se le asigna el prefijo "e", como sucede por ejemplo en el 'e-learning' o la 'e-economía'; igualmente, la articulación de procesos de relación entre pares, se integran en el marco conceptual del "P2P"; etcétera. 
etnográfico contrastar los "temas" destacados con las dimensiones estructurales de la sociedad donde residen los grupos investigados (siguiendo con el ejemplo, aquí cabría hablar de una e-sociedad, cibersociedad, sociedad digital, etc.). Ese procedimiento forma parte del marco de intenciones de la etnografía y ha hecho posible denominaciones como las de etnografía urbana o etnografía audiovisual, que referencian un aspecto estructural sometido a convención dentro del marco semántico que rodea al objeto de estudio. Lo distintivo en este caso tiene lugar cuando se produce un desmarque conceptual respecto de las condiciones de posibilidad —en términos de affordances (Wellman et al., 2003) — que ofrecen las tecnologías digitales, asignándole sustantividad a dimensiones que son meramente instrumentales ${ }^{11}$. Esa alteración conceptual puede afectar a varios niveles de una disciplina y condicionar tanto sus aspectos fundamentales como su marco de acción. Una de las consecuencias de esa confusión en la etnografía, es el sesgo hacia lo procedimental que ha caracterizado a las propuestas sobre su práctica en los nuevos contextos digitales ${ }^{12}$. Centrarse en aspectos instrumentales, como por ejemplo el análisis de datos digitales o las entrevistas mediadas tecnológicamente, lleva a dejar de lado otros aspectos de mayor interés epistémico, relacionados con el tipo de conocimiento que se espera alcanzar como resultado de la práctica metodológica, y que también pueden verse alterados por los cambios profundos que introduce "lo digital".

Uno de los posibles cambios significativos es el relacionado con la hibridación y su impacto en la configuración de las nuevas estructuras sociales. La hibridación afecta de un modo directo a una de las misiones centrales del etnógrafo, como es la de la "suministrar los contextos" (Turner, 1994) o, lo que es lo mismo, plasmar, reflejar y representar las formas de vida de los grupos objeto de análisis. Una sociedad híbrida es aquella en la que la sociabilidad — esto es, tanto el entramado de relaciones significativas que forman el contexto, como la construcción sociocultural a su alrededor - se produce en el marco de una serie de espacios digitales que interoperan con las dimensiones físicas de la realidad, permitiendo expandir la capacidad de acción de los sujetos en diferentes órdenes. Como ya se ha planteado, lo digital actúa como un amplificador de las posibilidades del mundo físico en sus aspectos estructurales. Y ello hace posible que surjan prácticas innovadoras que suceden en

${ }^{11}$ En el caso de la etnografía y los estudios cualitativos en general, en estos últimos años se han sucedido las investigaciones que ponen de manifiesto la materialidad de las prácticas sociales en foros virtuales, wikis, blogs o en redes como Facebook o Twitter. En esos y otros casos han sido frecuentes las reflexiones acerca de lo sustantivo de ciertos aspectos de forma que están en su mayoría circunscritos a la capacidad de las funcionalidades de una determinada aplicación —en el caso del 'software' —, o a la configuración de un dispositivo —en el caso del 'hardware'-. Conceptos como 'meme', hipertexto, hilo conversacional, 'timeline', RSS, muro, IRC, ROPO, etc., han sido tomados como elementos definitorios de prácticas estables vinculadas con la cultura digital, cuando en su mayoría son propios de un determinado marco de posibilidad definido a partir de la configuración (cambiante) de cada dispositivo y aplicación.

${ }^{12}$ Entre otras dimensiones del hacer etnográfico, ese componente procedimental se ha centrado especialmente en aspectos como la representación de las observaciones, el discurso narrativo empleado en el análisis de la "realidad" y en los métodos de observación e interpretación de lo observado/practicado por el etnógrafo. 
lugares intermedios constituidos en interfaces semiconductoras entre el mundo físico y el digital. Podría considerarse como anticipativa de este concepto la visión de McLuhan (1977) de los medios como "extensiones de nosotros mismos" y "extensiones de nuestros sentidos", si bien los últimos referentes están más próximos a las teorías del comportamiento y las de la elección social, citadas en el apartado anterior al referirnos a la aproximación de Sen.

La mayoría de los investigadores interesados en la etnografía virtual están familiarizados con el término "etnografía mixta", que se deriva a su vez de las formas de hacer etnografía multilocal (Marcus, 1995). Aplicada en escenarios de mediación digital, esa metodología combina el análisis de la información obtenida en el estudio de las prácticas sociales del mundo físico y las que tiene lugar en las pantallas. Pero con el tiempo, las concepciones acerca de "lo mixto", "lo múltiple" o lo "multisituado" han evolucionado hasta convertirse en constructos dotados de una mayor carga simbólica o representacional en relación con aquello que pretenden referenciar. La hibridación sociotécnica incorpora otra dimensión en este escenario, al poner de manifiesto la parcialidad del trabajo de campo localizado físicamente. Atendiendo a los presupuestos epistémicos de la etnografía, en un entorno híbrido sería parcial investigar únicamente las prácticas en la pantalla. Ahora, la multicontextualidad que permite plasmar la trama discursiva en el relato etnográfico y la ubicuidad geográfica donde se referencia una determinada cultura adquieren un nuevo significado. Esto es algo muy evidente en las situaciones intermedias entre lo social y lo técnico, que se dan particularmente en los entornos altamente conectados. Pero afecta de igual modo al conjunto de situaciones de análisis en la medida en que lo tecnosocial constituye un rasgo diferencial de las sociedades contemporáneas.

En efecto, el relato etnográfico es una de las dimensiones de la práctica etnográfica que se ven interpeladas directamente por las situaciones de hibridación. En los enfoques híbridos, la información procede de diferentes canales y planos superpuestos y su interpretación requiere combinar esas fuentes dotando al conjunto de un discurso integrador. En los modelos de investigación basados en la hibridación, el énfasis no está en la tecnología, los entornos, las prácticas o el componente material de las mismas cuando tienen lugar en ciberespacios. Esos elementos pueden constituirse en artefactos culturales y ser objeto de experimentación desde otros múltiples enfoques cualitativos. Pero cuando se trata de la práctica etnográfica, el eje analítico se traslada hacia los discursos que suceden en las interfaces, por lo que la trama se construye mediante el ensamblaje de elementos multinivel.

El ensamblaje es una técnica que permite configurar puntualmente, en un momento y un lugar determinados, una constelación de prácticas-ideas-personas-objetos dispersos pero que comparten principios comunes (Corsin, 2010). El momento y el lugar pueden tener una espacio-temporalidad muy abierta, por lo que para la etnografía resulta de especial interés la posibilidad de integrar en un mismo relato los espacios y los tiempos donde se plasman los productos culturales (híbridos) generados fragmentariamente en contextos de esta naturaleza.

El uso de técnicas como el ensamblaje en la construcción del relato etnográfico es de utilidad para investigar fenómenos socioculturales que transcurren en 
diferentes planos entrecruzados entre lo físico y lo digital. Además, su interés está también asociado con la necesidad de afrontar otros fenómenos, como la ubicuidad y lo transmediático, que pueden interferir en la factibilidad de la etnografía en situaciones de hibridación.

\section{Contextos ubicuos, cultura en red}

La consideración actual acerca de la tecno-cultura - esto es, el conjunto de realizaciones sociales derivadas de la intersección entre tecnología, cultura y sociedadha estado precedida de un conjunto heterogéneo de aproximaciones conceptuales a los nuevos mundos que surgen posibilitados por las tecnologías digitales. Algunas de las causas que explican esa diversidad de aproximaciones han sido citadas someramente en apartados anteriores ${ }^{13}$. Como hemos comentado, el interés en una adecuada aproximación conceptual en este terreno puede ser del interés de la etnografía por cuanto las tecnologías digitales introducen cambios sustantivos en la estructura social que pueden afectar a algunas bases epistémicas de la disciplina.

En la misma esfera conceptual de la hibridación, la ubicuidad constituye una dimensión analítica que explica la capacidad expansiva de lo digital que sucede cuando tecnologías y procesos sociales entran en resonancia. Un entorno ubicuo podría definirse como un tipo de escenario concreto para la práctica social cuya materialidad tiene un componente híbrido, ya que ocupa un lugar intermedio entre la realidad física y la virtual. La ubicuidad tiende a difuminar los límites entre ambas realidades, pero lo hace de un modo que facilita la convergencia en torno a nodos locales dotados de rasgos identitarios específicos (Monge y Contractor, 2003; Benkler, 2006). Lo que es de especial interés para los análisis cualitativos de la socio-cultura.

Comprender esa estructura es clave para las investigaciones basadas en el enfoque naturalista, puesto que hace referencia a los elementos que regulan no solo las prácticas sino también las condiciones de posibilidad en el "interior" de estos espacios. El componente híbrido de un entorno ubicuo se debe a que las relaciones en sus márgenes no transcurren enteramente deslocalizadas y descorporificadas, sino que surgen en el límite entre lo virtual y lo real. En el caso de la etnografía, un marco de acción de este tipo afecta de un modo particular al proceso de contextualización significativa y a la localización de los datos, ambas cuestiones claves en el relato etnográfico. Cuando el investigador reconstruye las acciones vividas durante el trabajo de campo, lo hace con el objetivo de dotar al relato de una visión de conjunto, holística, con la que atrapar la realidad y los datos materiales que son analizados. La explicación de las informaciones se da formando parte de una situación concreta que cobra sentido en una determinada estructura sociocultural. De manera que los datos materiales están dotados de un sentido local que puede reflejarse mediante la descripción densa, mientras que los aspectos estructurales remiten a un determinado patrón que enmarca la sociedad y las culturas analizadas. Las situaciones de ubicuidad

${ }^{13}$ De manera resumida, estas serían: en los estudios sociales, la proximidad de los análisis a las situaciones de práctica; en el caso de los estudios teóricos, el uso de modelos paradigmáticos analógicos; y en general, la constante transformación de las funcionalidades tecnológicas, que dificulta la validación de los hallazgos de investigación y las propuestas teorías. 
amplían ese marco interpretativo, que ahora posee varias "capas" analíticas. Lo que demanda recurrir a nuevas fuentes de información y técnicas de contraste y validación contextual para dotar de explicaciones válidas a los fenómenos estudiados.

Por otro lado, hablar de nodos ubicuos remite a un modelo de sociabilidad digital que posee una base estructural en forma de red. Llevado al ámbito sociocultural, ese componente reticular da lugar a nuevas manifestaciones de la cultura, que ahora pasa a ser una cultura en red (Echeverría, 2009).

En este punto cabe hacer una distinción entre la cultura digital (en red) y la tecnocultura, en un ejercicio que es próximo a la ya clásica disociación entre el ciberespacio como artefacto generador de culturas $-\mathrm{y}$, por tanto, susceptible de ser objeto de estudios etnográficos fragmentarios - y la cultura de Internet - el conjunto de prácticas que caracterizan los comportamientos y evidencias simbólicas producidas por sus "actores"- . Como se ha planteado anteriormente, al igual que cabe hablar de una cultura de Internet en un sentido restrictivo y acotada temporalmente en el desarrollo inicial de esa tecnología, también es propio hablar de tecnoculturas consideradas como resultantes de las operaciones realizadas con los objetos digitales accesibles solo a través de dispositivos de mediación tecnológicos. Lo oportuno de plantear esta visión procede de la diferente situación —en términos de condiciones de posibilidad - que surge con las estructuras reticulares para aquellos agentes generadores de cultura. Definidas de ese modo restrictivo, las tecnoculturas se refieren básicamente a un componente performativo del objeto digital. Sus ámbitos de acción dejan de lado la posibilidad de constituir artefactos mixtos, generados en el terreno intermedio de los flujos y cuya representatividad tiene sentido únicamente en ese territorio intermedio, interconectado e híbrido. Un ejemplo claro de esta situación es el análisis del uso de 'hashtags' en las prácticas discursivas públicas. Una convocatoria pública de adhesión a un determinado movimiento que emplea códigos lingüísticos basados en la sintaxis de la red social Twitter, es solo comprensible desde un gradiente intrepretativo que tenga en cuenta el componente mixto, físico y virtual, que impregna ese discurso social. Pues bien, es en ese sentido que las tecnoculturas, en su consideración mas abierta, no solo no se referirían únicamente a las culturas de los objetos digitales, sino que cobran su significación en el nuevo escenario expandiéndose y abarcando el basto campo de la sociabilidad híbrida en una forma de cultura en red.

En lo que al trabajo del etnógrafo se refiere, una de sus tareas centrales a desarrollar en los espacios donde se mezclan procesos tecno-sociales, consiste en desentrañar la ecología que resulta de las prácticas mediadas que suceden en diferentes planos de la realidad. Esto es porque la mediación digital dota a las nuevas ecologías de entornos de práctica donde no sólo se permite la producción de contenidos, sino también de los contextos. Esto es, los propios contextos se producen como consecuencia de las prácticas, que son las que construyen la sociabilidad a partir de las condiciones de posibilidad de los medios digitales ${ }^{14}$. Además, en el caso de

${ }^{14}$ Por ejemplo, un foro virtual se constituye en contexto a partir de la interacción de sus miembros; su existencia anterior es nula y se limita a las líneas de código y las conexiones entre 
las prácticas culturales, los contextos ubicuos permiten una relación novedosa del agente con el espacio (el mundo exterior) y con el lugar (el entorno socio-cultural próximo).

Lo anterior dibuja un escario basado en la convergencia de herramientas de mediación y en la ubicuidad de las estructuras culturales en múltiples localizaciones físicas y virtuales. Todo ello habilita formas de sociabilidad y de producción de cultura distribuidas en diferentes niveles. Formatos que impactan directamente en aspectos de fondo de interés para el etnógrafo y también en las metodologías empleadas para producir discursos científicos. Siguiendo con el hilo conductor del texto, a continuación nos detendremos en las nuevas narrativas basadas en prácticas socioculturales cruzadas (transmedia) y cómo éstas pueden ensamblarse dentro del relato etnográfico.

\section{Prácticas expandidas, narrativas transmedia}

Thomas (1993; citado por Hammersley y Atkinson, 1994: 220) hace la siguiente valoración referida al uso del hipertexto en las aplicaciones informáticas de análisis cualitativo de datos:

Al utilizar hipertextos, un investigador puede incluir no solo una descripción convencional del método de estudio, sino también gráficos (fotografías o segmentos de vídeo) y sonido para ilustrar o clarificar su procedimiento. Para los investigadores cualitativos el hipertexto puede resultar especialmente útil, pues podría incluir en él, en un disco que cabe en la palma de la mano, las anécdotas que ilustran conceptos, así como los segmentos reales de entrevistas que estructuran la información.

Como es sabido, el hipertexto es uno de los elementos constitutivos de la web. Aplicado en la edición de contenidos, permite generar discursos no lineales empleando simultáneamente diferentes soportes 'online' ${ }^{15}$. Thomas señala estos aspectos y su potencial para enriquecer el análisis de datos y la construcción del relato etnográfico, en lo que supone una de las primeras contribuciones de la digitalización al terreno de la investigación cualitativa. Pese a la pertinencia de sus reflexiones, estas fueron realizadas en un momento en el que las tecnologías digitales tan solo podían actuar acompañando el trabajo del etnógrafo. Su impacto social y, por tanto, la contribución más amplia a la creación del tejido social era muy menor. Ahora bien, en un escenario como el descrito, en el que las tecnologías ya no solo afectan a una parcela de la realidad situada tras la pantalla, sino que permean el entramado

dispositivos que lo harán posible una vez comience la acción.

${ }^{15}$ En su clásica aproximación, George Landow (1995) define al hipertexto como: "un texto compuesto de bloques de palabras (o de imágenes) electrónicamente unidos en múltiples trayectos, cadenas o recorridos en una textualidad abierta, eternamente inacabada." Entre las características fundamentales del hipertexto Landow destaca tres: (1) la intertextualidad, (2) la polifonía y (3) el descentramiento. La consecuencia inmediata de producir un relato mediante hipertexto es la fragmentación: el orden lineal — es decir un orden lógico-causal— pierde su funcionalidad y viene a ser sustituido por una estructura multilineal. Para una reflexión más reciente sobre la aportación del hipertexto en el ámbito de las humanidades, véase Rodríguez (2006). 
social, redefiniéndolo y amplificándolo, el análisis debe necesariamente ampliar su alcance y llegar a cuestionarse sobre los cambios que tienen lugar en el género narrativo sobre el que se construye el relato etnográfico. En un espacio de hibridación, las propiedades del hipertexto se proyectan a los otros muchos órdenes de la sociabilidad, dando paso a una sucesión de acontecimientos conectados que tienen lugar en múltiples escenarios. Esas conexiones permiten definir un ecosistema caracterizado por la generación de acciones producidas en un cruce de medios, y por consiguiente, con un alto componente transmediático.

Un ejemplo de cómo las nuevas narrativas transmedia afectan al relato etnográfico puede verse en el trabajo de investigación "Occupy Research", sobre el movimiento "OccupyWallStreet / OccupyTogether" (véase, http://occupyresearch. wikispaces.com/). El diseño de investigación consiste en un conjunto de técnicas y procesos analíticos. Todo ello presentado sobre la base de un documento digital de gestión colaborativa que actúa como "pegamento" de las actuaciones que se llevarán a cabo. A su vez, los fenómenos objeto de estudio no poseen una entidad definida, sino que afectan a diferentes procesos sociales y están "presentes" en una multitud ambivalente de contextos de práctica: abiertos y cerrados, digitales y analógicos, desde lógicas grassroot e institucionales. Otros dos ejemplos significativos son los del uso del ciber-mapeado colaborativo en procesos de obtención de datos multisituados y con un componente globalizado (véase el proceso de documentación de redadas racistas en la ciudad de Madrid en una investigación sobre grupos neonazis, http://goo.gl/5NbS7); o las nuevas formas de practicar investigación acción participativa que emplean narrativas transmedia (en el caso que se plantea, mediante el uso del hashtag \#opEuribor) y el apoyo popular (crowd) para generar la documentación (véase, http://opeuribor.es/our-research/).

En estas situaciones la abundancia de espacios, tipos de datos, tecnologías, métodos y variaciones de métodos, enfoques analíticos, etc. hace que las etnografías posibles se hagan densas, por lo que es preciso considerar nuevas formas de producir relatos. De acuerdo con la finalidad analítica de este texto, nos detendremos en dos de esas nuevas formas por considerarlas de interés en el marco de redefinición epistémica planteada.

En primer lugar, según se ha dicho, uno de los principales cometidos del etnógrafo es el de ensamblar los procesos significativos que tienen lugar en la práctica social. En las nuevas estructuras multinivel esa forma de integración se convierte en un elemento central, ya que permite reordenar las descripciones procedentes del trabajo de campo y generar un argumentario capaz de identificar y comprender los procesos culturales. A su vez, la técnica el ensamblaje va adquiriendo ciertas singularidades que la diferencian de otros procesos analíticos para el estudio de datos cualitativos. Así, en los casos en los que el fenómeno analizado abarca múltiples capas de sociabilidad cruzadas por diferentes tecnologías, el ensamblaje se da en el espacio y el tiempo de integración del contexto que lo contiene, donde convergen personas, componentes virtuales, simbólicos, etc.

La principal dificultad para aplicar esta técnica en situaciones expandidas es la de gestionar grandes cantidades de información. Habitualmente, el etnógrafo hace 
uso de instrumentos que le permiten registrar las evidencias de rasgos socioculturales en los contextos de práctica. También aplica ciertas técnicas analíticas tanto procedentes de la lingüística, como de los métodos sociales inductivos. Pues bien, a este bagaje se le suman ahora otros instrumentos y métodos que aportan a la investigación elementos positivos similares a los señalados por Thomas (1993) en referencia al hipertexto ${ }^{16}$. Como reflexión solo cabe apuntar que si bien el uso de estas nuevas técnicas en una monografía etnográfica tiende a enriquecer los hallazgos y la calidad representativa del relato - especialmente, cuando se pretende mantener la dimensión holística del discurso, aún partiendo de puntos de convergencia ubicuos-, esto sucede a costa de sofisticar el proceso de un modo que resulta evidente.

El segundo componente que destacaremos aquí en relación con la construcción del relato etnográfico en situaciones de hibridación sociotécnica, se refiere a la generación de la corriente discursiva (storytelling) generada en torno a las descripciones y los argumentos. Cuando las observaciones tienen lugar en ambientes multinivel y las narrativas de los sujetos ocurren en múltiples escenarios (físicos y virtuales), el discurso generado también se sitúa en una dimensión transmediática.

En este punto resulta pertinente introducir una reflexión sobre la "cualidad" de las narraciones digitales no-lineales y multinivel. Desde una aproximación cognitivista, una narración, y la personalidad que se construye a través de una narración, transmite la perspectiva individual de "nosotros-en-el-mundo". Esta perspectiva incluye la comprensión individual acerca del funcionamiento de las causas y los efectos de nuestro comportamiento. A su vez, las narrativas también transmiten las redes causales que conforman la vida de los sujetos en formas que se ajustan a alguno de los prototipos narrativos que son comprensibles por todo el mundo, en el contexto donde suceden los acontecimientos. Por ello, para que pueda ser transmitida, la narración requiere ordenar temporalmente los acontecimientos más destacados así como dotarlos de contexto.

Según esta aproximación, en un primer momento las narrativas digitales en contextos abiertos, sin restricciones de orden temporal y causal, pudieran parecer psicológicamente inverosímiles como fuente de datos fiables. Sin embargo, la lógica de los entornos de hibridación y la narración transmedia hace posible la producción de ese tipo de secuencias narrativas más allá de la ortodoxia lineal.

Una narración transmedia remite a múltiples plataformas en las que cada elemento contribuye de manera única a crear un universo narrativo para el lector. El uso de diferentes plataformas crea diferentes "puertas de acceso" para sumergirse en la historia (Fleming, 2011; Pratten, 2010; Riedel, 2010). Las narrativas transmedia surgieron en el ámbito de las industrias culturales con la finalidad de emplearlas para proyectar historias figuradas más allá de los canales habituales, llegando así

${ }^{16}$ La principal técnica de gestión de datos cualitativos en entornos digitales abiertos es la analítica web. Su uso en ámbitos sociales se aplica sobre todo al análisis de tendencias. Recientemente, también desde la educación se ha comenzado a emplear para monitorizar el comportamiento de los estudiantes en ambientes online abiertos (Ferguson y Buckingham, 2011; Siemens, 2011). Para un ejemplo específico de uso de estas técnicas en el análisis de procesos relacionados con la cultura, véase Leetaru (2011). 
a más públicos mediante estrategias de marketing cruzadas. Pero las referencias, alusiones y conexiones intertextuales que forman parte de la complejidad narrativa de una secuencia transmedia, puede dar lugar también de diferentes formas en procesos donde el relato debe contener datos procedentes de fuentes mixtas, físicas y virtuales. Este es el caso de la etnografía, en donde su uso hace posible expandir el componente argumental del relato e incorporar a la construcción de la trama cultural nuevas dimensiones analíticas y formas de representarlas.

\section{Referencias bibliográficas}

\section{ALVAREZ, José Francisco}

2001 "Capacidades, libertades y desarrollo: Amartya Kumar Sen" en MÁIZ, R.(Comp.), Teorías politicas contemporáneas. Valencia: Tirant lo blanch, 381-396.

ALLEN, Christina

1996 "What's wrong with the 'Golden Rule'? Conundrums of conducting ethical research in cyberspace". Information Society, 12: 175-187.

ARDÉVOL, Elisenda; ESTALELLA, Adolfo; DOMÍNGUEZ, Daniel (Coords.)

2008 "La mediación tecnológica en la práctica etnográfica". Actas del XI Congreso de Antropología, San Sebastián: Ankulegi.

BEAULIEU, Anne

2004 "Mediating Ethnography: Objectivity and the Making of Ethnographies of the Internet". Social Epistemology, 18: 139-163.

BENKLER, Yochai

2006 The Wealth of Networks. How Social Production Transforms Markets and Freedom. New Haven \& London: Yale University Press.

BIELLA, Peter

1996 "Interactive media in anthropology: Seed and Earth - promise of rain". American Anthropologist, 98: 595-616.

BIJKER, Wiebe; LAW, John (Eds.)

1992 Shaping Technology / Building Society: Studies in Sociotechnical Change (Inside Technology). Massachusetts: MIT Press.

BURRELL, Jenna

2009 "The Field Site as a Network: A Strategy for Locating Ethnographic Research". Field Methods, 21(2):181-199.

CALLON, Michael

1986 "Some elements of a sociology of translation: Domestication of the scallops and the fishermen of St. Brieuc Bay.” En Law, J. (Ed.), Power, Action and Belief: A New Sociology of Knowledge? Londres: Routledge, 196-223.

CASTELLS, Manuel

2001 La galaxia Internet. Reflexiones sobre Internet, empresa y sociedad. Barcelona: Plaza \& Janés. 
CORSIN, Alberto

2010 “Zonas, objetos, ensamblajes”. Prototyping: how social experimentation works, http://www.prototyping.es/uncategorized/zonas-objetos-ensamblajes

DENZIN, Norman

1996 Interpretive Ethnography: Ethnographic Practices for the 21st Century. Thousand Oaks: Sage.

Di MAGGIO, Paul; HARGITTAI, Eszter; NEUMAN, W. Russel; ROBINSON, John P.

2001 “The Internet's Effects on Society". Annual Reviews of Sociology, 27: 307-336.

DIANI, Mario

2000 "Social Movement Networks Virtual and Real". Information Communication y Society, 3(3): 386-401.

DÍAZ DE RADA, Ángel

2010 "La lógica de la investigación etnográfica y la mediación computacional de la comunicación. Viejos problemas con un nuevo énfasis". Revista Chilena de Antropología Visual, 15: 40-57.

DICKS, Bella; MASON, Bruce

1998 "Hypermedia and Ethnography: Reflections on the Construction of a Research Approach”. Sociological Research Online, 3(3).

DOMÍNGUEZ, Daniel

2007 "Sobre la intención de la etnografía virtual”. Revista Electrónica Teoría de la Educación. Educación y Cultura en la Sociedad de la Información, 8(1), http:// dialnet.unirioja.es/servlet/articulo?codigo $=2284795$ yorden $=113536$ yinfo=link

DOMINGUEZ, Daniel; ÁLVAREZ, José Francisco

2012 "Redes sociales y espacios universitarios. Conocimiento e innovación abierta en el espacio iberoamericano del conocimiento". Revista de Universidad y Sociedad del Conocimiento (RUSC), 9(1), http://rusc.uoc.edu/ojs/index.php/rusc/article/view/ v9n1-dominguez-alvarez/v9n1-dominguez-alvarez

ECHEVERRÍA, Javier

2009 "Cultura digital y memoria en red". Arbor: Ciencia, pensamiento y cultura, 737: 559-567.

ESTALELLA, Adolfo

2005a “Anatomía de los blogs. La jerarquía de lo visible". TELOS, Revista de Comunicación e Innovación, 65, http://sociedadinformacion.fundacion.telefonica. com/telos/articulocuaderno.asp@idarticulo=9yrev=65.htm

2005b "Filtrado colaborativo: la dimensión sociotécnica de una comunidad virtual". UOC Papers, 1, http://www.uoc.edu/uocpapers/1/dt/esp/estalella.pdf

ESTALELLA, Adolfo; ARDÉVOL, Elisenda

2010 "Internet: instrumento de investigación y campo de estudio para la antropología visual”. Revista Chilena de Antropología Visual, 15: 1-21. 
FARNELL, Brenda; HUNTLEY, Joan

1995 "Ethnography Goes Interactive". Anthropology Today, 111: 7-10.

FERGUSON, Rebecca; BUCKINGHAM, Simon

2011 "Learning Analytics to Identify Exploratory Dialogue within Synchronous Text Chat." Proc. 1st International Conference on Learning Analytics y Knowledge. Feb. 27-Mar 1, Banff, http://oro.open.ac.uk/28955

FLEMING, Laura

2011 National Writing Project. Transmedia Collection. California: Digital Is \& University of California, http://issuu.com/larfleming/docs/transmedia_collection? mode=embedylayout $=\mathrm{http} \% 3 \mathrm{~A} \% 2 \mathrm{~F} \% 2 \mathrm{Fskin}$.issuu.com $\% 2 \mathrm{Fv} \% 2 \mathrm{Flight} \% 2 \mathrm{Flayout}$. xmlyshowFlipBtn=true

GÓMEZ, Edgar

2007 Las metáforas de Internet. Barcelona: UOC.

GRAY, Chris Hables (Ed.)

1995 The Cyborg Handbook. Nueva York: Routledge.

HAMMERSLEY, Martyn; ATKINSON, Paul

1994 Etnografía. Métodos de investigación. Barcelona: Paidós.

HARAWAY, Donna J.

1991 Simians, Cyborgs, and Women: Reinventing Nature. Nueva York: Routledge.

HERRING, Susan C.

2004 "Slouching toward the ordinary: Current trends in computer-mediated communication". New Media and Society, 6: 26-36.

HINE, Christine

2000 Virtual Ethnography. Londres: Sage.

2007 "Connective ethnography for the exploration of e-science". Journal of ComputerMediated Communication, 12.

HINE, Christine (Ed.)

2005 Virtual Methods. Issues in Social Research on the Internet. Oxford: Berg.

HOWARD, Philip

2002 "Network ethnography and the hypermedia organization: new organizations, new media, new methods". New Media and Society, 4: 551-575.

ILLINGWORTH, Nicola

2001 "The Internet Matters: Exploring the Use of the Internet as a Research Tool". Sociological Research Online, 6.

JONES, Steven G. (Ed.)

1998 Cybersociety 2.0: Revising Computer-Mediated Communication and Society. Thousand Oaks: Sage. 
KATZ, James E.; RICE, Ronald E.; ASPDEN, Philip

2001 "The Internet 1995-2000: Access, Civic Involvement, and Social Interaction". American Behavioral Scientist, 45(3): 405-419.

LANDOW, George

1995 Hipertexto. La convergencia de la teoría crítica contemporánea y la tecnología. Barcelona: Paidós.

LATOUR, Bruno

1994 On Technical Mediation - Philosophy, Sociology, Genealogy. Common Knowledge, 3: 29-64.

LEETARU, Kaley Hannes

2011 "Culturomics 2.0: Forecasting Large-Scale Human Behavior Using Global News Media Tone in Time and Space". First Monday, (16)9, http://www.uic.edu/htbin/ cgiwrap/bin/ojs/index.php/fm/article/view/3663/3040

LEUNG, Linda

2005 Virtual Ethnicity. Race, Resistance and the World Wide Web. Ashgate: Aldershot.

MARCUS, George E.

1995 "Ethnography in/of the World System. The Emergence of Multi-Sited Ethnography". Annual Review of Anthropology, 24: 95-117.

MARKHAM, Annete N.

1998 Life Online. Researching real experience in virtual space. Londres: Altamira Press.

MAYANS, Joan

2001 “Género confuso: género Chat". TEXTOS de la CiberSociedad, 1, http://www.cibersociedad.net/textos/articulo.php?art=22

2002 Género chat. O cómo la etnografía puso un pie en el ciberespacio. Barcelona: Gedisa.

MCLUHAN, M

1977 La comprensión de los medios como extensions del hombre. México: Diana.

MEYROWITZ, Joshua

1985 No sense of Place: the Impact of Electronic Media on Social Behavior. Nueva York: Oxford University Press.

MILLER, Daniel; SLATER, Don

2000 The Internet. An Ethnographic Approach. Nueva York: Berg.

MONGE, Peter R; CONTRACTOR, Noshir

2003 Theories of Communication Networks. Oxford: Oxford University Press.

PRATTEN, Robert

2010 Transmedia Storytelling: Getting Started. WorkBook Project, http://workbookproject.com/culturehacker/2010/07/07/transmedia-storytelling-getting-started/ 
RHEINGOLD, Howard

1993 The Virtual community. Homesteading in the electronic frontier. Nueva York: Harper Perennial.

RIEDEL, Christian

2010 "Transmedia Storytelling - An Introduction." Conference on Hamburg Writers Group, December 15, http://www.slideshare.net/mindcaffeine/ transmedia-storytelling-6176436

RODRÍGUEZ, Jaime Alejandro

2006 "Qué es un (hiper)texto”. En Chartier, R. (ed.), ¿Qué es un texto? Madrid: Círculo de Bellas Artes, 87-100.

SIEMENS, George

2011 “Open Learning Analytics: A proposal”. Elearningspace, http://www.elearnspace. org/blog/2011/12/10/open-learning-analytics-a-proposal/

SILVER, David

2004 "Internet/cyberculture/digital culture/new media/fill-in-the-blank studies". New Media and Society, 6.

THOMAS, J.

1993 "Catching up to the cyber age" Writing Sociology, 1, 2: 1-3.

TURKLE, Sherry

1995 Life on the Screen. Identity in the Age of the Internet. Nueva York: Simon and Schuster.

TURNER, Stephen

1994 The Social Theory of Practices, Tradition, Tacit Knowledge and Presuppositions. Oxford: Policy.

VELASCO, Honorio; DÍAZ DE RADA, Ángel

2006 La lógica de la investigación etnográfica. Madrid: Trotta.

WELLMAN, Barry; QUAN-HAASE, Anabel; BOASE, Jeffrey; et al.

2003 "The Social Affordances of the Internet for Networked Individualism." JCMC, 8(3), http://jcmc.indiana.edu/vol8/issue3/wellman.html

WOO, Han

2003 "Hyperlink Network Analysis: A New Method for the Study of Social Structure on the Web". Connections, 25: 49-61.

WOOLGAR, Steve

1996 "Technologies as Cultural Artifacts." En MALCOLM, P. y. WILLIAM H.D, (Eds.), Information and Communication Technologies. Visions and Realities. Oxford: Oxford University Press, 87-102. 